\title{
On the trends in kinetic energies of secondary ions produced by polyatomic ion bombardment ${ }^{\text {ts }}$
}

\author{
Igor V. Veryovkin ${ }^{\mathrm{a}, *}$, Sergey F. Belykh ${ }^{\mathrm{b}}$, Annemie Adriaens ${ }^{\mathrm{c}}$, \\ Alexander V. Zinovev ${ }^{\mathrm{a}}$, Freddy Adams ${ }^{\mathrm{b}}$ \\ ${ }^{a}$ Materials Science Division, Argonne National Laboratory, Argonne, IL 60439, USA \\ ${ }^{\mathrm{b}}$ Department of Chemistry, University of Antwerp (UIA), B-2610 Antwerp (Wilrijk), Belgium \\ ${ }^{\mathrm{c}}$ Department of Analytical Chemistry, Ghent University, B-9000 Ghent, Belgium
}

Available online 6 May 2004

\begin{abstract}
Kinetic energies of $\mathrm{Ta}_{n}{ }^{+}$and $\mathrm{Nb}_{n}{ }^{+}$ions $(1 \leq n \leq 10)$ sputtered from pure Ta and $\mathrm{Nb}$ targets by $6 \mathrm{keV}$ per atom $\mathrm{Au}^{-}, \mathrm{Au}_{2}{ }^{-}$and $\mathrm{Au}_{3}{ }^{-}$primary ions have been compared after energy spectra of these ions were recalibrated using a newly developed data processing algorithm. Most probable and mean energies were determined for the sputtered ions, and their energy spectra were converted into distributions over inverse velocities. Distributions obtained for Ta and $\mathrm{Nb}$ displayed many similarities and no principal differences. The only differences seen were due to the difference in masses of the elements. On the other hand, strong differences were observed between results for atomic and diatomic sputtered ions and those for larger sputtered cluster ions (with more than seven atoms). The comparison of atomic and polyatomic bombardment showed dramatic changes in emission of atomic and diatomic sputtered species, and almost no differences for larger sputtered clusters.
\end{abstract}

(C) 2004 Elsevier B.V. All rights reserved.

Keywords: Molecular beams interactions with solids; Secondary ion mass spectrometry

\section{Introduction}

Polyatomic ion bombardment generated strong interest in secondary ion mass spectrometry (SIMS) community in the last decade [1] due to the recognition of such advantages of this regime as enhanced signals of secondary ions improving detection limits

\footnotetext{
This work is supported by the US Department of Energy, BESMaterials Sciences, under Contract No. W-31-109-ENG-38 and by the Belgian Federal Services for Scientific, Technical and Cultural Affairs (DWTS/SSTC) of the Prime Minister's Office through IUAP-5.

* Corresponding author. Tel.: +1-630-252-4657; fax: +1-630-252-9555.

E-mail address: verigo@anl.gov (I.V. Veryovkin).
}

and reduced primary ion ranges improving depth resolution. Despite a non-additive enhancement of secondary ion signal under polyatomic ion bombardment has been long known [2], its mechanism remains poorly understood. To date, it is unclear what actually increases, the total sputtering yield or the ionization probability of sputtered neutrals [3].

In this work, we compare results of polyatomic bombardment of two different materials with similar physical and chemical properties but different masses, $\mathrm{Nb}$ and $\mathrm{Ta}$, and show how additional information on the process of their sputtering can be extracted from experimentally measured kinetic energy distributions of secondary atomic and cluster ions. These targets were selected under the assumption that their similarity 
would result in similar charged state formation processes, while the difference in masses would influence processes of their sputtering. TRIM [4] simulations estimate sputtering yields for bombardment with $6 \mathrm{keV}$ $\mathrm{Au}$ ions at $45^{\circ}$ incidence angle to be 4.6 and 7.8 ; and projected ranges of ions to be 36 and $24 \AA$ for $\mathrm{Nb}$ and Ta, respectively.

Development of a new data processing procedure for the calibration of kinetic energies of secondary ions [5] allowed us to determine their absolute energies and thus to conduct a more accurate comparison of the energy distributions for the same secondary ions sputtered by different projectiles. Therefore we revisited raw experimental data for previously published in $[6,7]$ kinetic energy spectra of $\mathrm{Ta}$ and $\mathrm{Nb}$ atomic and cluster ions sputtered by $6 \mathrm{keV}$ per atom $\mathrm{Au}^{-}, \mathrm{Au}_{2}{ }^{-}$ and $\mathrm{Au}_{3}{ }^{-}$primary ions and reprocessed them using the new procedure. As a part of this data re-examination, a conversion of the experimental kinetic energy distributions of secondary ions into distributions of velocities and inverse velocities was performed. Trends revealed by this effort are discussed below in view of differences and similarities between $\mathrm{Nb}$ and Ta bombarded by atomic and polyatomic ions.

\section{Experimental and data processing procedures}

A magnetic sector SIMS instrument described in more details in [7] was equipped with a cluster ion source [8] that generated polyatomic ions $\mathrm{Au}_{m}{ }^{-}$ ( $m=1,2,3)$ by sputtering gold targets with $\mathrm{Cs}^{+}$ions. The procedure used in measurements of kinetic energy spectra of secondary ions was described in details elsewhere [6,7]. The energy resolution of the experimental apparatus (its response function) was estimated by the measurement of kinetic energy spectra of thermal ions of alkali metals, $\mathrm{Na}^{+}$and $\mathrm{K}^{+}$, formed on the hot sample surface in absence of ion bombardment. The full widths at half maximums of these energy spectra were determined to be about $5.5 \mathrm{eV}$ at $2000 \mathrm{~V}$ nominal acceleration voltage.

To compare energy spectra measured under bombardment by different projectiles, a new calibration procedure has been developed [5] that determined and aligned zeroes of energy scales of all measured spectra. A simple linear approximation of near-zero energy regions of such spectra and extrapolation of these straight lines down to zero intensity as described in [9] is often used for spectra alignment/comparison purposes. Unfortunately, this procedure cannot produce absolute energies of secondary ions. Our new algorithm aimed at more accurate determination of the absolute energies. To this end, we modelled how true energy distributions are "smeared" by the measurements with a finite response function. This was done by a comparison between model kinetic energy spectra of sputtered $\mathrm{Ta}$ and $\mathrm{Nb}$ atoms calculated using Sigmund-Thompson formula [10,11] and smeared distributions calculated as an integral of convolution of these model spectra with the function found to be the best approximation for the energy spectra of thermal ions. The comparison revealed that maximums of smeared/convolved model spectra were shifted towards higher energies from those of the original model spectra. Moreover, our analysis of data from $[6,7]$ showed that if all fitting parameters are locked except the one that defines position of the maximum then the same fitting function, as used for the measured energy spectra of thermal ions, could well approximate not only near-zero region of the smeared model spectrum but also such regions of all experimentally measured kinetic energy spectra of secondary ions. We set the initial value of the fitting parameter responsible for the position of the maximum to that for the smeared model spectra and then determined new values of this parameter for all experimentally measured kinetic energy spectra. Shifting experimental spectra by the difference between the initial value and the new ones calibrated their energy scales so that all low energy portions of the spectra appeared to be on one line determined for the smeared model spectra [5].

\section{Results and discussion}

Experimental raw data from [6,7] were recalibrated as described above. Then additional data processing of kinetic energy distributions $\mathrm{d} N / \mathrm{d} E$ was performed to determine most probable energies and mean kinetic energies calculated as

$\bar{E}=\frac{\int E(\mathrm{~d} N / \mathrm{d} E) \mathrm{d} E}{\int(\mathrm{d} N / \mathrm{d} E) \mathrm{d} E}$

Results of these calculations are presented in Fig. 1a and $b$. 


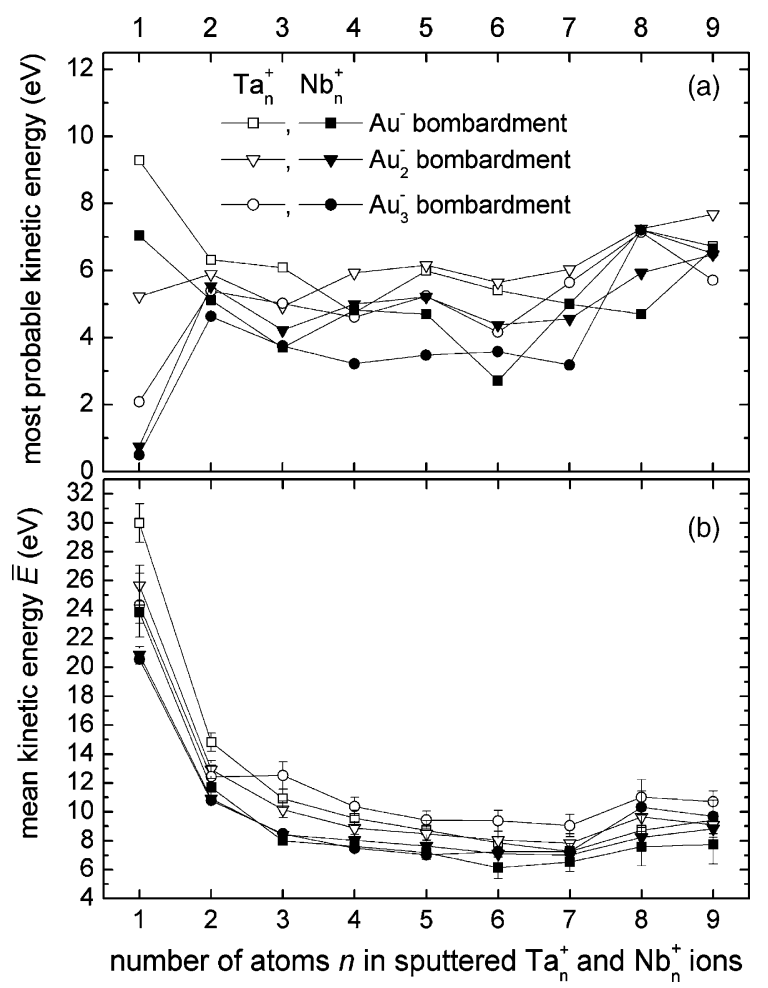

Fig. 1. Most probable kinetic energies (a) and mean kinetic energies (b) determined from kinetic energy distributions $\mathrm{d} N / \mathrm{d} E$ of $\mathrm{Nb}_{n}{ }^{+}$and $\mathrm{Ta}_{n}{ }^{+}$secondary ions $(n=1-9)$ sputtered by $6 \mathrm{keV}$ per atom $\mathrm{Au}_{m}{ }^{-}(m=1,2,3)$ primary ions.

The comparison between dependencies obtained for $\mathrm{Nb}$ and Ta reveals qualitative similarities and nonsignificant quantitative differences such as slightly lower energies for $\mathrm{Nb}$ compared to the corresponding data for Ta.

The comparison between different primary ions leads to more complex picture, as follows. One can see in Fig. 1 that for both $\mathrm{Nb}$ and Ta the most probable energies and the mean energies of secondary ions reveal significant differences for atomic ions, slight differences for diatomic ions and practically no differences (within the experimental confidence intervals) for larger cluster ions.

The most striking trend seen in Fig. 1 is that the change from atomic to polyatomic projectiles causes decrease in kinetic energies of sputtered atoms (strongly pronounced) and dimers (less pronounced). The most probable energy of sputtered atomic ions is defined both by that energy of sputtered neutral atoms and by their ionization probability, which can be also energy dependent [10,11]. For neutral sputtered atoms, their most probable kinetic energy depends on the energy distribution of recoil atoms in the collision cascade and on the height of the surface energy barrier. In the linear collision cascade model of sputtering, the height of this barrier is equal to the surface binding energy often approximated by the sublimation energy of the sputtered material $[4,10,11]$. To date, there is no consensus in the literature on what value should be used to estimate the surface barrier for sputtering with polyatomic ions. It is unclear in what extent the linear cascade model is applicable for this case when the material properties, including the height of the surface barrier, can substantially change due to the high density of energy deposited by a polyatomic projectile into the nearsurface region, which might generate there electronic excitation. On the other hand, these changes might cause non-linear development of collision cascades in the solid, their overlapping etc., which may modify energy distributions of recoil atoms. Under the assumption that the ionization probability does not dramatically changes, kinetic energy distributions of sputtered atomic ions can be considered as "sensors" of changes in the sputtering process. In this case, the increased contribution of low energy atomic ions in the spectra we observe under polyatomic bombardment might indicate that both the surface barrier became lower and that the energy distribution of recoils was enriched with low energy atoms. In terms of the sputtering yield, lower barrier corresponds to a higher yield, while more recoils with low energy, at the same barrier height, correspond to a lower yield. As significant increase in signals of sputtered species is observed under polyatomic bombardment, it seems reasonable to attribute the decrease in the most probable energies to lowering of the surface barrier. Using the terminology of the linear cascade model (and keeping in mind its questionable applicability as discussed above) one can conclude that our experiments might reveal the decrease of the surface binding energy first hypothesized for polyatomic ion bombardment in [12].

It is commonly accepted in the ion sputtering related science that the velocity of sputtered species is more fundamental than the energy in determining their ionization probability [13]. Therefore, to get 
insights in the ionization process, we converted kinetic energy distributions of sputtered ions $\mathrm{d} N / \mathrm{d} E$ into distributions of velocities $\mathrm{d} N / \mathrm{d} v$ and distributions of inverse velocities $\mathrm{d} N / \mathrm{d}\left(v^{-1}\right)$ using the Jacobians corresponding to these transformations:

$\frac{\mathrm{d} N}{\mathrm{~d} v}=\frac{\mathrm{d} N}{\mathrm{~d} E(v)} \frac{\mathrm{d} E(v)}{\mathrm{d} v}=\frac{\mathrm{d} N}{\mathrm{~d} E(v)} M v$,

$\frac{\mathrm{d} N}{\mathrm{~d}\left(v^{-1}\right)}=\frac{\mathrm{d} N}{\mathrm{~d} E\left(v^{-1}\right)} \frac{\mathrm{d} E\left(v^{-1}\right)}{\mathrm{d}\left(v^{-1}\right)}=\frac{\mathrm{d} N}{\mathrm{~d} E\left(v^{-1}\right)}\left[-\frac{M}{\left(v^{-1}\right)^{3}}\right]$,

where $M$ is the mass of the sputtered particle. Multiplying inverse velocities by distance produces time necessary to move over that distance. Distributions over such times, $\mathrm{d} N / \mathrm{d} t$, are proportional to the distributions in inverse velocities, $\mathrm{d} N / \mathrm{d}\left(v^{-1}\right)$. If a crystal lattice parameter is chosen as such a distance, then the distributions $\mathrm{d} N / \mathrm{d} t$ will indicate time required for sputtered atoms and clusters to move away from the surface to the distance of one lattice parameter. For both $\mathrm{Nb}$ and $\mathrm{Ta}$, the lattice parameter is $a=3.3 \AA$. In models of charged state formation in sputtering [13], distances of this order are often considered as the characteristic ones for electron exchange processes occurring between the departing sputtered atom, or cluster, and the surface. Estimating the amount of time the sputtered species spend in the region of interaction with the surface may give us clues to the recognition of processes on the surface that contribute to the charge formation.

Three sets of distributions, $\mathrm{d} N / \mathrm{d} v, \mathrm{~d} N / \mathrm{d}\left(v^{-1}\right)$, and $\mathrm{d} N / \mathrm{d} t$, were calculated for $\mathrm{Nb}$ and $\mathrm{Ta}$ for all combinations of primary/secondary ions shown in Fig. 1. As with kinetic energy distributions $\mathrm{d} N / \mathrm{d} E$ above, in order to compare so many different curves, we calculated mean values of velocity, inverse velocity, and time needed to travel one lattice parameter:

$\bar{v}=\frac{\int v(\mathrm{~d} N / \mathrm{d} v) \mathrm{d} v}{\int(\mathrm{d} N / \mathrm{d} v) \mathrm{d} v}$,

$\bar{v}^{-1}=\frac{\int v^{-1}\left(\mathrm{~d} N / \mathrm{d}\left(v^{-1}\right)\right) \mathrm{d}\left(v^{-1}\right)}{\int\left(\mathrm{d} N / \mathrm{d}\left(v^{-1}\right)\right) \mathrm{d}\left(v^{-1}\right)}$,

$\bar{t}=\frac{\int t(\mathrm{~d} N / \mathrm{d} t) \mathrm{d} t}{\int(\mathrm{d} N / \mathrm{d} t) \mathrm{d} t}$.

Fig. 2 shows the dependencies of mean inverse velocities $\bar{v}^{-1}$ and mean times $\bar{t}$ on the number of atoms $n$ in the detected sputtered ion.

The most noticeable feature in Fig. 2 is an almost exact likelihood of two groups of near-parabolic curves for $\mathrm{Nb}$ and $\mathrm{Ta}$. It seems reasonable to assume that the electron exchange processes for species sputtered from $\mathrm{Nb}$ and $\mathrm{Ta}$, materials with similar electron

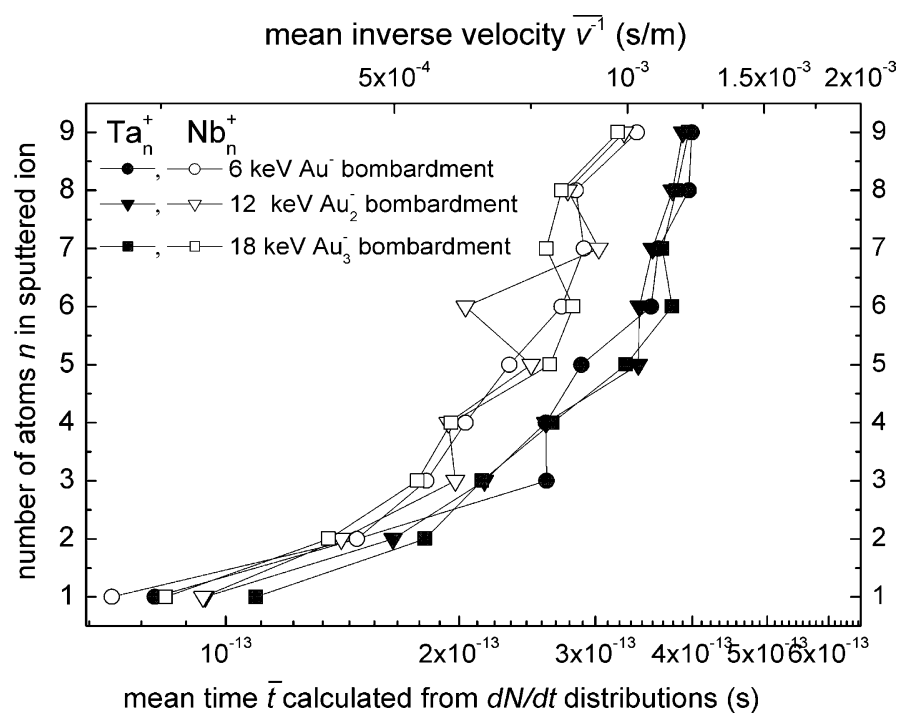

Fig. 2. Mean inverse velocities and mean times needed to travel the distance of one lattice parameter determined $\mathrm{Nb}_{n}{ }^{+} \operatorname{and~Ta}_{n}{ }^{+}(n=1-9)$ secondary ions sputtered by $6 \mathrm{keV}$ per atom $\mathrm{Au}_{m}{ }^{-}(m=1,2,3)$ primary ions. 
properties, occur similarly. If any characteristic minimal velocity (or maximal time) can be attributed to such a charge state formation process as a measure of successful emission in the ionized state then one can expect these parameters to have similar values for these two elements. What is important to notice in Fig. 2 is that the shift between groups of curves corresponding to $\mathrm{Nb}$ and to Ta is originating not from minor differences in kinetic energies seen in Fig. 1 but from about factor of two difference in the masses of the elements. This may suggest that what we see, especially the near-parabolic behaviour of the curves in the range of sputtered cluster ions, is the manifestation of regularities of the sputtering process which is energy dependent rather that that for the ionization which is velocity dependent.

The other trend seen in Fig. 2 is that for both elements and for all three projectiles $\mathrm{Au}_{m}{ }^{-}(m=1$, 2,3 ) values of $\bar{v}^{-1}$ and $\bar{t}$ become undistinguishable at $n=3$. In the region of sputtered atoms, dimers and trimers these values increase while $m$ increases, i.e., secondary ions produced by molecular bombardment are slower, on average. The whole picture suggests that polyatomic ion bombardment of both $\mathrm{Ta}$ and $\mathrm{Nb}$ does not cause any dramatic changes in the ionization probability of sputtered species as compared to atomic ion bombardment.

\section{Conclusion}

1. Results we obtained for secondary ions suggest that lowering of surface energy barrier under polyatomic ion bombardment may cause most of the yield enhancement observed in our experiments. On the other hand, the very fact that the experiments were carried out for sputtered ions and provided no data on sputtered neutrals prevents us from completely excluding from our consideration ionization enhancements for sputtered atomic species.
2. No strong dependence on the size of the projectile is observed for most probable and mean kinetic energy of sputtered cluster species. In fact, the energies of clusters of the same element appear to be about the same, and the energies of $\mathrm{Nb}$ clusters, on average, are about $1 \mathrm{eV}$ lower than those for Ta.

3. Mean inverse velocities (and derived from their values mean times needed to travel the distance of one lattice parameter) show similar dependencies on the number of atoms $n$ for $\mathrm{Nb}_{n}{ }^{+}$and $\mathrm{Ta}_{n}{ }^{+}$ions. The analysis does not reveal any visible influence of the velocity dependent charged state formation process on these dependencies. The influence of the energy dependent sputtering process is more evident. As a hypothesis, one can suggest that the ionization processes occurred much earlier than the characteristic times of our experiments.

\section{References}

[1] G. Gillen, A. Fahey, Appl. Surf. Sci. 203 (2003) 209.

[2] H.H. Andersen, Mat. Fys Medd. Dan. Vid. Selsk. 43 (1993) 127.

[3] S.F. Belykh, V.V. Palitsin, I.V. Veryovkin, A. Adriaens, F. Adams, Nucl. Instr. Meth. B 203 (2003) 164.

[4] J.F. Ziegler, J.P. Biersack, U. Littmark, The Stopping and Range of Ions in Solids, Pergamon Press, New York, 1985.

[5] I.V. Veryovkin, S.F. Belykh, A. Adriaens, F. Adams, Nucl. Instr. Meth. B, in press.

[6] S.F. Belykh, U.Kh. Rasulev, A.V. Samartsev, I.V. Veryovkin, Nucl. Instr. Meth. B 137 (1998) 773.

[7] S.F. Belykh, B. Habets, U.Kh. Rasulev, A.V. Samartsev, L.V. Stroev, I.V. Veryovkin, Nucl. Instr. Meth. B 164-165 (2000) 809.

[8] S.F. Belykh, R.N. Evtukhov, Ju.N. Lysenko, U.Kh. Rasulev, Rev. Sci. Instr. 63 (4) (1992) 2458.

[9] H. Gnaser, Phys. Rev. B. 54 (1996) 17141.

[10] W. Hofer, in: R. Behrish, K. Wittmaack (Eds.), Sputtering by Particle Bombardment, III, Springer, Berlin, 1991, p. 14.

[11] G. Betz, K. Wien, Int. J. Mass Spectrom. Ion Proc. 140 (1994) 1.

[12] S.S. Johar, D.A. Thompson, Surf. Sci. 90 (1979) 310.

[13] M. Yu, in: R. Behrish, K. Wittmaack (Eds.), Sputtering by Particle Bombardment, III, Springer, Berlin, 1991, p. 91. 